\title{
APPROXIMATIONS OF VERY WEAK SOLUTIONS TO BOUNDARY-VALUE PROBLEMS*
}

\author{
MARTIN BERGGREN ${ }^{\dagger}$
}

\begin{abstract}
Standard weak solutions to the Poisson problem on a bounded domain have squareintegrable derivatives, which limits the admissible regularity of inhomogeneous data. The concept of solution may be further weakened in order to define solutions when data is rough, such as for inhomogeneous Dirichlet data that is only square-integrable over the boundary. Such very weak solutions satisfy a nonstandard variational form $(u, v)=G(v)$. A Galerkin approximation combined with an approximation of the right-hand side $G$ defines a finite-element approximation of the very weak solution. Applying conforming linear elements leads to a discrete solution equivalent to the text-book finite-element solution to the Poisson problem in which the boundary data is approximated by $L^{2}$-projections. The $L^{2}$ convergence rate of the discrete solution is $O\left(h^{s}\right)$ for some $s \in(0,1 / 2)$ that depends on the shape of the domain, assuming a polygonal (two-dimensional) or polyhedral (three-dimensional) domain without slits and (only) square-integrable boundary data.
\end{abstract}

Key words. finite-element methods, very weak solution, transposition, rough data

AMS subject classifications. 35J05, 35J20, 65N30

DOI. $10.1137 /$ S0036142903382048

1. Introduction. Applications such as optimal control, inverse problems, and shape optimization sometimes call for boundary data that are rougher than the theory for elliptic or parabolic boundary-value problems routinely assumes. This note addresses numerical issues in the presence of rough boundary data.

We restrict the discussion to a simple case, the Poisson equation with inhomogeneous Dirichlet conditions,

$$
\begin{aligned}
-\Delta u=f & \text { in } \Omega, \\
u=g & \text { on } \Gamma,
\end{aligned}
$$

where $\Omega$ is a bounded domain in $\mathbb{R}^{2}$ or $\mathbb{R}^{3}, \Gamma$ the domain boundary, and $f$ and $g$ are given data. Integration by parts yields that smooth solutions to (1) satisfy

$$
\int_{\Omega} \nabla u \cdot \nabla v d x=\int_{\Omega} f v d x
$$

for each smooth $v$ vanishing on $\Gamma$. The "standard" weak solution to (1), the basis for finite-element discretizations, satisfies variational expression (2) with $u$ and $v$ being elements in certain subspaces of $H^{1}(\Omega)$, the Sobolev space of order one.

Which type of boundary data $g$ makes sense to specify in (1)? The answer depends on which type of functions we accept as being solutions. For standard weak solutions, it is necessary that $g$ can be extended continuously into a function in $H^{1}(\Omega)$. Such extensions are not always possible - there are even continuous functions $g$ on the

*Received by the editors March 26, 2003; acceped for publication (in revised form) October 6, 2003; published electronically June 4, 2004. This research was supported in part by the Computer Science Research Institute at Sandia National Laboratories. Sandia is a multiprogram laboratory operated by Sandia Corporation, a Lockhead Martin Company, for the United States Department of Energy under contract DE-AC04-94AL85000.

http://www.siam.org/journals/sinum/42-2/38204.html

$\dagger$ Department of Information Technology, Uppsala University, Box 337, SE-751 05 Uppsala, Sweden (Martin.Berggren@it.uu.se). 
boundary $\Gamma$ that do not extend continuously. The situation is even worse for nonsmooth data. For instance, no function with jump discontinuities on the boundary can be extended continuously into a function in $H^{1}(\Omega)$.

However, even weaker solutions to system (1) than the standard weak solution relax the requirements on $g$. Integrating (2) by parts once more reveals that solutions to (1) satisfy

$$
-\int_{\Omega} u \Delta v d x=-\int_{\Gamma} g \frac{\partial v}{\partial n} d \Gamma+\int_{\Omega} f v d x
$$

for each smooth $v$ vanishing on $\Gamma$. The boundary integral on the right-hand side of expression (3) now makes sense for $g$ being merely square-integrable, as long as the normal derivative of $v$ is also square-integrable on the boundary. Variational expression (3) - in a version made precise in section 4-is the basis for defining very weak solutions to the Poisson equation with boundary data being no more than squareintegrable. Compared to the variational form (2), the nonstandard variational form (3) relaxes the regularity requirement on $u$ and $g$ at the price of higher regularity requirements on $v$, a particular example of the method of transposition, treated in great generality in the classic three-volume treatise of Lions and Magenes [15].

This note considers a numerical approximation based on the Lions-type variational expression (3) and proves optimal-order convergence rates for linear, conforming elements. To the best of my knowledge, this approach to discretizing problems with rough boundary data has not previously been reported in the literature. Previous analysis of problems with rough data has concerned other discretization approaches.

Babuška [1] defines weak solutions using a generalized Lax-Milgram lemma, a version that would apply, for instance, to the form (3) in the case of full elliptic regularity and a smooth boundary. (Because of lack of regularity, we cannot directly use his method here.) He considers finite-element approximations of the Poisson problem based on the standard variational form (2) with homogeneous boundary data and proves error bounds that cover also very weak solutions such as Green's functions.

French and King [8] analyze a parabolic initial-boundary-value problem for convex domains in $\mathbb{R}^{2}$. Using temporal averaging combined with spatial $L^{2}$-projections for the boundary data, they prove error estimates for a standard finite-element approximation in space, combined with the backward Euler scheme in time, of a very weak solution to the parabolic problem.

In the context of an optimal-control problem for a second-order elliptic equation on convex domains in $\mathbb{R}^{2}$, French and King [7] introduce a standard finite-element approximation and show that it converges to the very weak solution defined by transposition. Another contribution in this direction is by Bramble and King [2]. They consider elliptic problems on smooth, curved domains in $\mathbb{R}^{2}$, and their error estimates hold also for rough boundary data.

In all articles cited above, $L^{2}(\Gamma)$-projections approximate the rough boundary data, which allows the use of the standard variational form (2). In contrast, this article uses the Lions-type variational form (3) as the basis for discretization. Thus, the variational form in the discretization is identical to the one used to define the (very) weak solution, and projection of the data is not needed. Nonetheless, a perhaps surprising result of this article (Theorem 5.2) is that the discrete solution obtained with the current approach is equivalent to the standard finite-element approximation combined with $L^{2}(\Gamma)$-projections of the inhomogeneous data. This observation removes 


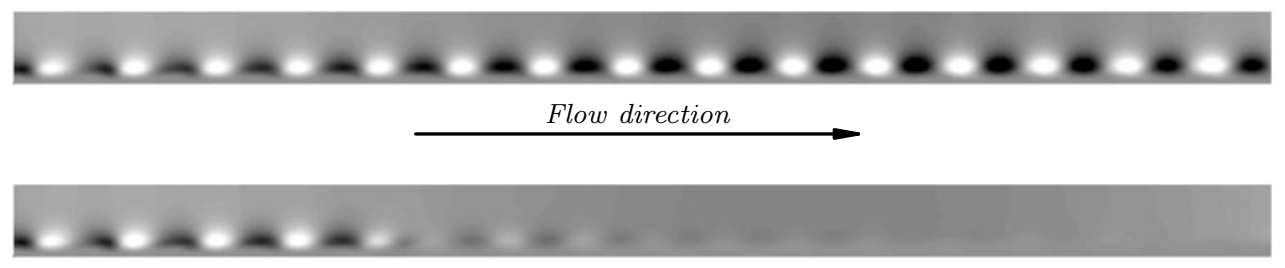

FIG. 1. Wall-normal velocity disturbance levels depicting Tollmien-Schlichting waves in a boundary layer over a flat plate. The lower picture shows the result after applying boundary control at a portion of the wall.

some of the arbitrariness of the standard method with data projections, showing its equivalence with a systematic scheme based on the Lions-type variational form.

Another difference from previous work is that the analysis below covers both two and three space dimensions with polygonal or polyhedral boundaries, without assuming convexity of the domain. The analysis in the articles cited above is restricted to two dimensions and assumes convex, polygonal domains.

Solutions as weak as the ones considered here have interest beyond mathematical curiosity. Applications in which very weak solutions appear naturally are boundary control and inverse problems $[4,9,14]$. The rest of the presentation starts with a short outline of this background in section 2. Section 3 reviews some fundamentals, notation, and the approximation properties that are needed, setting the stage for a more precise description in section 4 of the Poisson-equation solution based on transposition. Section 5 introduces a finite-element approximation of the very weak solution, proving convergence rates and equivalence with the "standard" approximation with projection of the data.

2. Background. Scientific and technical reasons prompt the need for controlling the behavior of solutions to partial differential equations, for instance through boundary action. In fluid-dynamics applications, the object is typically to manage the evolution of disturbances. Figure 1 depicts a boundary layer with evolving TollmienSchlichting waves, the most unstable disturbance according to linear stability theory. The pictures are snapshots from numerical solutions of the unsteady, incompressible Navier-Stokes equations in three space dimensions. A suitable blowing and suction at a portion of the lower boundary dramatically damps the disturbances, as shown in the lower picture of Figure 1. Chevalier et al. [4] report details of this and several similar computations. The blowing and suction is the numerical solution to a nonlinear optimization problem that minimizes the disturbances in the domain over a space of admissible controls. The admissible controls are boundary conditions with no more regularity a priori than being square-integrable functions on a portion of the lower boundary and during a finite time interval. This is a weaker regularity requirement on the boundary condition than needed for weak solutions of the Navier-Stokes equations.

The same regularity concern is an issue also for simpler problems that are easier to analyze. Consider, for instance, the following inverse problem for the Poisson equation (1). Given a function $z$ defined in a subdomain $\omega \subset \Omega$, find the boundary condition $g$ that yields $u=z$ in $\omega$. Thinking of (1) as a model for steady heat conduction in a homogeneous, isotropic solid, the inverse problem consists of estimating the temperature $g$ on the boundary given measurements in the interior (in $\omega$ ). 
The inverse problem above is only solvable for a very restricted class of targets $z$. Perhaps the easiest way of getting around this restriction is to solve a linear least-squares problem and minimize the objective function

$$
J(g)=\frac{\epsilon}{2} \int_{\Gamma} g^{2} d \Gamma+\frac{1}{2} \int_{\omega}(u-z)^{2} d x,
$$

where $\epsilon>0$ is a (Tikhonov) regularization parameter, included to prevent $g$ from becoming unbounded. The classical exposition of optimal control problems of this sort is the book by Lions [14]. A newer review by Glowinski and Lions [9] covers also numerical aspects.

To minimize $J$ among all $g \in L^{2}(\Gamma), u$ needs to be well defined and squareintegrable for each $g \in L^{2}(\Gamma)$. However, the standard variational form (2) of the Poisson equation requires also the derivatives of $u$ to be elements in $L^{2}(\Omega)$, a property that does not hold for all $g \in L^{2}(\Omega)$. Including also derivatives (possibly fractional) of $g$ along the boundary in the regularization term of $J$ fixes this problem. However, the derivatives of $g$ complicate a numerical solution of the control problem and introduce an extra smoothing, which may be unwanted, of the $g$ that minimizes $J$.

There are also other reasons to prefer $L^{2}$ norms. For instance, for studies of stability and transition in fluid mechanics, the customary measure of the "size" of velocity quantities is expressed as $L^{2}$-like norms, because of the connection to the kinetic energy of the fluid (Schmid and Henningson [16]).

\section{Preliminaries.}

3.1. Notation, function spaces. Consider an open, bounded, and connected domain $\Omega$ in $\mathbb{R}^{2}$ or $\mathbb{R}^{3}$ with a Lipschitz boundary $\Gamma$; that is, the boundary is locally the graph of a Lipschitz function (for details see Definition 1.2.1.1 in Grisvard [11], for instance). We denote by $H^{s}(\Omega)$ the Sobolev space of order $s$ on $\Omega$. When $s$ is a nonnegative integer, $H^{s}(\Omega)$ is the space in which each function and all its (weak) partial derivatives up to order $s$ are square-integrable over $\Omega$. We use the convention $H^{0}(\Omega)=L^{2}(\Omega)$ and $H^{0}(\Gamma)=L^{2}(\Gamma)$. Introducing a norm containing integrals over the domain, as in Definition 1.2.1 in Grisvard [12], generalizes the definition of $H^{s}(\Omega)$ to any real positive $s$. An alternative generalization uses interpolation of Hilbert spaces, as in section 2 of Chapter 1 in Lions and Magenes [15, Volume 1]. Brenner and Scott [3, Theorem 12.2.7] provide a proof that the spaces generated in these two ways are equivalent when the boundary is Lipschitz.

The trace $\gamma v$ of a function $v \in H^{s}(\Omega)$ generalizes to Sobolev spaces the restriction $\left.v\right|_{\Gamma}$ of a smooth function $v$ to the boundary. Unfortunately, the presence of "edges" and "corners" on a nonsmooth boundary complicates the trace concept compared to the case when the boundary is smooth. Nevertheless, it follows from Theorem 1.5.1.2 in Grisvard [11] that for $s \in(1 / 2,1]$, each function $v$ in $H^{s}(\Omega)$ has a well-defined trace $\gamma v$ in the Sobolev space $H^{s-1 / 2}(\Gamma)$, and that there exists a $C>0$ such that

$$
\|\gamma v\|_{s-1 / 2, \Gamma} \leq C\|v\|_{s} \quad \forall v \in H^{s}(\Omega) .
$$

Expression (4) uses the notation $\|\cdot\|_{s, \Gamma}$ for norms on $H^{s}(\Gamma)$. Analogously to $H^{s}(\Omega)$, integrals over the Lipschitz boundary $\Gamma$ define a norm on $H^{s}(\Gamma)$ as long as $s \in[0,1]$ (section 1.3.3 in Grisvard [11]). Restricting the domain of integration, we may also define norms $\|\cdot\|_{s, \Gamma_{i}}$ on open subsets $\Gamma_{i}$ of $\Gamma$.

The closure of $C_{0}^{\infty}(\Omega)$, the infinitely differentiable functions with compact support in $\Omega$, with respect to the norm in $H^{s}(\Omega)$ forms a subspace denoted $H_{0}^{s}(\Omega)$. In 
particular it holds that

$$
H_{0}^{1}(\Omega)=\left\{v \mid v \in H^{1}(\Omega), \gamma v=0\right\} ;
$$

that is, $H_{0}^{1}(\Omega)$ is the subspace of functions in $H^{1}(\Omega)$ with zero trace.

Negative norms are defined by

$$
\|v\|_{-s}=\sup _{w \in H_{0}^{s}(\Omega) \backslash\{0\}} \frac{1}{\|w\|_{s}} \int_{\Omega} v w d x, \quad s>0 .
$$

This norm can be used to define $H^{-s}(\Omega)$, a space of distributions on $\Omega$ strictly larger than $L^{2}(\Omega)$ (for instance, the space $H^{-1}(\Omega)$ can then be identified with the dual space of $H_{0}^{1}(\Omega)$ ). However, we will need the norm (5) only for estimates of functions $v \in L^{2}(\Omega)$.

The "dual" to definition (5),

$$
\|w\|_{s}=\sup _{\left.v \in L^{2}(\Omega)\right) \backslash\{0\}} \frac{1}{\|v\|_{-s}} \int_{\Omega} v w d x
$$

holds for any $w \in H_{0}^{s}(\Omega)$. Yosida [18, Chapter III, section 10] provides a detailed proof for $s=1$, but the arguments are unchanged for any $s>0$. From definition (6) one immediately obtains the Cauchy-Schwarz-like inequality

$$
\int_{\Omega} v w d x \leq\|v\|_{-s}\|w\|_{s}
$$

Similarly,

$$
\|g\|_{-s, \Gamma_{i}}=\sup _{h \in H_{0}^{s}\left(\Gamma_{i}\right) \backslash\{0\}} \frac{1}{\|h\|_{s, \Gamma_{i}}} \int_{\Gamma_{i}} g h d \Gamma
$$

defines negative norms on open subsets $\Gamma_{i}$ of $\Gamma$. Again, we will apply this norm only on functions $g \in L^{2}\left(\Gamma_{i}\right)$. Let $g \in L^{2}(\Gamma)$ and $h \in C_{0}^{\infty}\left(\Gamma_{i}\right) \backslash\{0\}$ be given. Extending $h$ by zero on $\Gamma \backslash \Gamma_{i}$, we see that

$$
\frac{1}{\|h\|_{s, \Gamma_{i}}} \int_{\Gamma_{i}} g h d \Gamma=\frac{1}{\|h\|_{s, \Gamma}} \int_{\Gamma} g h d \Gamma .
$$

Noting that the extended $h$ is in $H_{0}^{s}\left(\Gamma_{i}\right)$ as well as in $H_{0}^{s}(\Gamma)$, and taking supremum, it follows that

$$
\|g\|_{-s, \Gamma_{i}} \leq\|g\|_{-s, \Gamma}
$$

Throughout the following, $C$ denotes a positive constant, independent of the choice of functions and, later, of the mesh parameter $h$. However, adhering to a customary abuse of notation, the actual value of $C$ may change, even within the same chain of inequalities.

3.2. Regularity of the Poisson problem with homogeneous boundary conditions. Let $\Omega$ be a polyhedral domain in $\mathbb{R}^{3}$ or a polygonal domain in $\mathbb{R}^{2}$. We require the domain to be Lipschitz, which excludes domains with slits. Given $v \in L^{2}(\Omega)$, there is a unique $z \in H_{0}^{1}(\Omega)$ such that

$$
\int_{\Omega} \nabla z \cdot \nabla w d x=\int_{\Omega} v w d x \quad \forall w \in H_{0}^{1}(\Omega)
$$


The regularity properties of solutions to (10) are crucial in the development below. In fact, the possibility of defining the very weak solutions is a consequence of the fact that the regularity is better than merely $z \in H_{0}^{1}(\Omega)$.

Indeed, if the boundary is smooth, the additional regularity $z \in H^{2}(\Omega)$ holds. This is still true for polygonal or polyhedral boundaries if the domain is convex. The regularity is reduced, however, in the vicinity of nonconvex portions of polygonal or polyhedral boundaries. Grisvard [12] proves precise regularity results (Theorem 2.4.3 for the two-dimensional case and Corollary 2.6.7 for the three-dimensional case), stating that there exists an $\epsilon \in(0,1 / 2]$, which depends on the shape of the domain, such that solutions to (10) are actually in $H^{3 / 2+\epsilon}(\Omega)$. The following estimate will be needed.

Theorem 3.1. There exist an $\epsilon \in(0,1 / 2]$ and $a C>0$ such that the solution $z \in H_{0}^{1}(\Omega)$ to (10) satisfies

$$
\|z\|_{3 / 2+\epsilon-s} \leq C\|v\|_{-s} \quad \forall v \in L^{2}(\Omega)
$$

for each $s \in[0,1]$.

Proof. By the regularity result quoted above, the closed-graph theorem yields that there exists an $\epsilon \in(0,1 / 2]$ such that

$$
\|z\|_{3 / 2+\epsilon} \leq C\|v\|_{0} \quad \forall v \in L^{2}(\Omega) .
$$

Using the notation

$$
\||\nabla w|\|_{0}^{2}=\int_{\Omega}|\nabla w|^{2} d x
$$

(10) implies that

$$
\frac{1}{\||\nabla w|\|_{0}} \int_{\Omega} \nabla z \cdot \nabla w d x=\frac{1}{\||\nabla w|\|_{0}} \int_{\Omega} v w d x
$$

for each nonzero $w \in H_{0}^{1}(\Omega)$. Taking the supremum yields that

$$
\begin{aligned}
\|z\|_{1 / 2+\epsilon} & \leq C\|z\|_{1} \leq C\||\nabla z|\|_{0} \\
& =C \sup _{w \in H_{0}^{1}(\Omega) \backslash\{0\}} \frac{1}{\||\nabla w|\|_{0}} \int_{\Omega} \nabla z \cdot \nabla w d x \\
& =C \sup _{w \in H_{0}^{1}(\Omega) \backslash\{0\}} \frac{1}{\||\nabla w|\|_{0}} \int_{\Omega} v w d x \leq C\|v\|_{-1} \quad \forall v \in L^{2}(\Omega),
\end{aligned}
$$

where the second and the last inequality follow from the fact that the seminorm $\||\nabla z|\|_{0}$ is equivalent to $\|z\|_{1}$ for $z \in H_{0}^{1}(\Omega)$. Estimates (12) and (13) imply that the linear mapping $v \mapsto z$ is bounded from $L^{2}(\Omega)$ into $H^{3 / 2+\epsilon}$ as well as from $H^{-1}$ into $H^{1 / 2+\epsilon}(\Omega)$. Estimate (11) then follows by operator interpolation of the mapping $v \mapsto z$.

We will also need expressions for the boundary flux associated with the solution $z$ to (10) and the regularity properties of the boundary flux. Let us first assume full elliptic regularity, that is, $z \in H^{2}(\Omega) \cap H_{0}^{1}(\Omega)$. Integration by parts of the product $-\phi \Delta z$ then yields that the boundary flux $\partial z / \partial n$ satisfies

$$
-\int_{\Gamma} \frac{\partial z}{\partial n} \phi d \Gamma=\int_{\Omega} v \phi d x-\int_{\Omega} \nabla z \cdot \nabla \phi d x \quad \forall \phi \in H^{1}(\Omega) .
$$


If, moreover, the boundary is smooth, the boundary flux is an element in $H^{1 / 2}(\Gamma)$. On polyhedral boundaries, however, the discontinuity of boundary normals complicates the definition of boundary-flux spaces. Nevertheless, the integration-by-parts property (14) holds also for Lipschitz domains in $\mathbb{R}^{n}$ as long as $z \in H^{2}(\Omega) \cap H_{0}^{1}(\Omega)$; it follows from Proposition 5.1.6 in Brenner and Scott [3], for instance. The following theorem proves a similar expression for less regular $z$.

THEOREM 3.2. There are an $\epsilon \in(0,1 / 2]$ and a $C>0$ such that, associated with any $v \in L^{2}(\Omega)$ and corresponding solution $z \in H_{0}^{1}(\Omega)$ to (10), there exists a unique $\lambda \in L^{2}(\Gamma)$ satisfying

$$
-\int_{\Gamma} \lambda \phi d \Gamma=\int_{\Omega} v \phi d x-\int_{\Omega} \nabla z \cdot \nabla \phi d x \quad \forall \phi \in H^{1}(\Omega)
$$

and the estimates

$$
\begin{aligned}
\|\lambda\|_{0, \Gamma} & \leq C\|v\|_{0}, \\
\|\lambda\|_{\epsilon-s, \Gamma_{i}} & \leq C\|v\|_{-s} \quad \text { for } i=1, \ldots, I \text { and } \forall s \in[0, \epsilon) .
\end{aligned}
$$

Proof. By assumption, the boundary of $\Omega$ can be written $\Gamma=\cup_{i=1}^{I} \bar{\Gamma}_{i}$, where each (open and bounded) $\Gamma_{i}$ is a planar polygon or a line segment embedded in $\mathbb{R}^{3}$ and $\mathbb{R}^{2}$, respectively. Let $n_{i}$ denote the (constant) outward-directed unit normal associated with each polygonal surface (or line segment) $\Gamma_{i}$. By Theorem 3.1, we know that there is an $\epsilon \in(0,1 / 2]$ such that, for each $v \in L^{2}(\Omega)$ supplied to (10), the solution satisfies $z \in H^{3 / 2+\epsilon-s} \forall s \in[0,1]$. Differentiation is a continuous operator from $H^{\alpha}(\Omega)$ into $H^{\alpha-1}(\Omega)$ as long as $\alpha \neq 1 / 2$ [11, Theorem 1.4.4.6]. Thus, for each $i=1, \ldots, I$,

$$
\frac{\partial z}{\partial n_{i}}=n_{i} \cdot \nabla z
$$

resides in $H^{1 / 2+\epsilon-s}(\Omega)$ since $3 / 2+\epsilon-s \neq 1 / 2$ for any $\epsilon \in(0,1 / 2]$ and $s \in[0,1]$. By further restricting $s$, we can apply the trace theorem for Lipschitz boundaries (Grisvard [11, Theorem 1.5.1.2]) to obtain the bounds, for $s \in[0, \epsilon), i=1, \ldots, I$,

$$
\left\|\frac{\partial z}{\partial n_{i}}\right\|_{\epsilon-s, \Gamma} \leq C\left\|\frac{\partial z}{\partial n_{i}}\right\|_{1 / 2+\epsilon-s} \leq C\|z\|_{3 / 2+\epsilon-s}
$$

where the second inequality follows from the above-mentioned continuity of differentiation.

Now, for $i=1, \ldots, I$, we define $\lambda_{i} \in L^{2}(\Gamma)$ as

$$
\lambda_{i}= \begin{cases}\frac{\partial z}{\partial n_{i}} & \text { on } \Gamma_{i}, \\ 0 & \text { on } \Gamma \backslash \Gamma_{i},\end{cases}
$$

and $\lambda \in L^{2}(\Gamma)$ as

$$
\lambda=\sum_{i=1}^{I} \lambda_{i} .
$$

The definition of $\lambda$, together with inequality (17) and Theorem 3.1, yields estimate (16). 
Noting that $\lambda$ in effect is a weak representation for $\partial z / \partial n$, we will prove the integration-by-parts formula (15) by approximating $z$ by smooth functions. Indeed, since $C_{0}^{\infty}(\Omega)$ is dense in $H^{3 / 2+\epsilon}(\Omega) \cap H_{0}^{1}(\Omega)$, there exists a sequence $\left\{\zeta_{n}\right\}_{n=1}^{\infty} \subset C_{0}^{\infty}(\Omega)$ such that

$$
\zeta_{n} \rightarrow z \quad \text { in } H^{3 / 2+\epsilon}(\Omega)
$$

as $n \rightarrow \infty$. It follows from inequality (17) that

$$
\frac{\partial \zeta_{n}}{\partial n_{i}} \rightarrow \lambda_{i} \quad \text { in } L^{2}\left(\Gamma_{i}\right)
$$

as $n \rightarrow \infty$.

Forming $-\Delta \zeta_{n}$, multiplying by $w \in H_{0}^{1}(\Omega)$, integrating by parts, and noting that strong convergence in $H^{3 / 2+\epsilon}(\Omega) \cap H_{0}^{1}(\Omega)$ implies convergence in $H_{0}^{1}(\Omega)$, it follows from (19) and (10) that

$$
\begin{aligned}
\int_{\Omega} w\left(-\Delta \zeta_{n}\right) d x & =\int_{\Omega} \nabla w \cdot \nabla \zeta_{n} d x \rightarrow \int_{\Omega} \nabla w \cdot \nabla z d x \\
& =\int_{\Omega} w v d x \quad \forall w \in H_{0}^{1}(\Omega)
\end{aligned}
$$

as $n \rightarrow \infty$. Since $H_{0}^{1}(\Omega)$ is dense in $L^{2}(\Omega)$, expression (21) yields that

$$
-\Delta \zeta_{n} \rightarrow v \text { weakly in } L^{2}(\Omega) .
$$

Let $\phi \in H^{1}(\Omega)$. Integration by parts yields

$$
\begin{aligned}
\int_{\Omega} \phi\left(-\Delta \zeta_{n}\right) d x & =-\int_{\Gamma} \frac{\partial \zeta_{n}}{\partial n} \phi d \Gamma+\int_{\Omega} \nabla \phi \cdot \nabla \zeta_{n} d x \\
& =-\sum_{i=1}^{I} \int_{\Gamma_{i}} \frac{\partial \zeta_{n}}{\partial n_{i}} \phi d \Gamma+\int_{\Omega} \nabla \phi \cdot \nabla \zeta_{n} d x .
\end{aligned}
$$

Letting $n \rightarrow \infty$, it follows from (18), (20), (21), and (22) that expression (23) converges to

$$
\int_{\Omega} \phi v d x=-\int_{\Gamma} \lambda \phi d \Gamma+\int_{\Omega} \nabla \phi \cdot \nabla z d x
$$

which proves that the $\lambda$ defined in expression (18) satisfies expression (15). Finally, since $\lambda$ depends linearly on $v \in L^{2}(\Omega)$, estimate (16) also provides uniqueness of $\lambda$ for each given $v \in L^{2}(\Omega)$.

3.3. Approximation properties. Let us now triangulate the polygonal or polyhedral domain $\Omega$ and introduce a mesh parameter $h>0$ that characterizes the triangulation. We assume nondegenerate meshes [3, Def. 4.4.13]; that is, there is a limit to how "thin" the tetrahedral may become as the mesh is refined. Denote by $V^{h}$ the space of continuous functions that are linear on each triangle or tetrahedron in the mesh, and denote by $V_{0}^{h}$ the subspace of functions in $V^{h}$ vanishing on $\Gamma$. We have $V^{h} \subset H^{1}(\Omega)$ and $V_{0}^{h} \subset H_{0}^{1}(\Omega)$. The restriction to $\Gamma$ of functions in $V^{h}$ is denoted $\gamma V^{h}$. We also define $M^{h}$ as the space of all functions $v^{h} \in V^{h}$ that vanish at each mesh point in the strict interior of the domain. We have that $V^{h}=M^{h} \oplus V_{0}^{h}$; that 
is, each function in $V^{h}$ is the sum of unique functions in $M^{h}$ and $V_{0}^{h}$. Also note that each function $g_{h} \in \gamma V^{h}$ uniquely extends to a function $\widehat{g}_{h} \in M^{h}$ that equals $g_{h}$ at the boundary but vanishes at all nodes in $\Omega$. This extension property is useful when solving inhomogeneous boundary-value problems: Given an approximation $g_{h} \in \gamma V^{h}$ of the boundary data $g$, extend $g_{h}$ to $\widehat{g}_{h} \in M^{h}$, write the solution $u_{h} \in V_{h}$ as $u_{h}=u_{h, 0}+\widehat{g}_{h}$, where $u_{h, 0} \in V_{0}^{h}$, and solve for $u_{h, 0}$.

An interpolation operator $\Pi_{h}$ from $H^{s}(\Omega)$ into $V^{h}$ characterizes the approximation properties of $V^{h}$. If $s>d / 2$, where $d$ is the space dimension, it follows from the Sobolev embeddings that $\Pi_{h}$ can simply be chosen as the linear interpolator of function values at the nodes of the triangulation. However, we need to consider small values of $s$, so pointwise values may not be well defined. It is therefore appropriate to choose the Scott and Zhang interpolator [17], which uses a local averaging to generate nodal values. This interpolator yields optimal-order estimates, and the averaging is constructed to preserve piecewise-polynomial boundary conditions, a property that Lemma 5.3 exploits.

The following approximation properties hold for $V^{h}$ : There exists a constant $C>0$ such that, for all $h>0$,

$$
\begin{array}{lll}
\left\|v-\Pi_{h} v\right\|_{1} & \leq C h^{s}\|v\|_{1+s} & \forall v \in H^{1+s}(\Omega), s \in[0,1] \\
\left\|v-\Pi_{h} v\right\|_{0} & \leq C h^{s}\|v\|_{s} & \forall v \in H^{s}(\Omega), s \in[0,2] .
\end{array}
$$

Standard textbooks, such as Ciarlet [5], prove these properties for integral values of $s$. Scott and Zhang [17] supply a proof for the particular case of the above-mentioned $\Pi_{h}$. Operator-interpolation arguments, discussed by Brenner and Scott [3, Chapter 12], for instance, extend the estimates to intermediate real numbers $s$.

We also need to approximate functions defined on the boundary. Recall that the domain is polyhedral or polygonal, so $\Gamma=\cup_{i=1}^{I} \bar{\Gamma}_{i}$, where each $\Gamma_{i}$ is an open planar polygon or an open line segment that does not overlap any other $\Gamma_{i}$. The space $\gamma_{i} V^{h}$ of traces of function in $V^{h}$ on $\Gamma_{i}$ is a space of continuous, piecewise-linear functions on the triangles (or intervals) of $\Gamma_{i}$. We may thus define an interpolation operator $\Pi_{h}^{\gamma_{i}}$ from $H^{s}\left(\Gamma_{i}\right)$ into $\gamma_{i} V^{h}$ with properties analogous to $\Pi_{h}$,

$$
\left\|g-\Pi_{h}^{\gamma_{i}} g\right\|_{0, \Gamma_{i}} \leq C h^{s}\|g\|_{s, \Gamma_{i}} \quad \forall g \in H^{s}\left(\Gamma_{i}\right), s \in[0,2] .
$$

Another type of approximation in $V^{h}, \gamma V^{h}$, and $\gamma_{i} V^{h}$ are the $L^{2}$-projections, that is, the functions $P_{h} v \in V^{h}, P_{h}^{\gamma} g \in \gamma V^{h}$, and $P_{h}^{\gamma_{i}} g \in \gamma_{i} V^{h}$ satisfying

$$
\begin{array}{rlrl}
\int_{\Omega} P_{h} v w_{h} d x & =\int_{\Omega} v w_{h} d x & & \forall w_{h} \in V^{h} \\
\int_{\Gamma} P_{h}^{\gamma} g \varphi_{h} d \Gamma & =\int_{\Gamma} g \varphi_{h} d \Gamma & \forall \varphi_{h} \in \gamma V^{h}, \\
\int_{\Gamma_{i}} P_{h}^{\gamma_{i}} g \varphi_{h} d \Gamma & =\int_{\Gamma_{i}} g \varphi_{h} d \Gamma & \forall \varphi_{h} \in \gamma_{i} V^{h}, i=1, \ldots, I,
\end{array}
$$

which are well defined for each $v \in L^{2}(\Omega)$ and $g \in L^{2}(\Gamma)$. The $L^{2}$-projections produce the discrete functions that minimize the $L^{2}$ error. 
For any $g \in L^{2}\left(\Gamma_{i}\right)$ and $\psi \in H_{0}^{s}\left(\Gamma_{i}\right)$, we have

$$
\begin{aligned}
& \int_{\Gamma_{i}}\left(g-P_{h}^{\gamma_{i}} g\right) \psi d \Gamma=\int_{\Gamma_{i}}\left(g-P_{h}^{\gamma_{i}} g\right)\left(\psi-P_{h}^{\gamma_{i}} \psi\right) d \Gamma \\
& \quad \leq\left\|g-P_{h}^{\gamma_{i}} g\right\|_{0, \Gamma_{i}}\left\|\psi-P_{h}^{\gamma_{i}} \psi\right\|_{0, \Gamma_{i}} \leq\left\|g-\Pi_{h}^{\gamma_{i}} g\right\|_{0, \Gamma_{i}}\left\|\psi-\Pi_{h}^{\gamma_{i}} \psi\right\|_{0, \Gamma_{i}} \\
& \quad \leq C\|g\|_{0, \Gamma_{i}} h^{s}\|\psi\|_{s, \Gamma_{i}} \quad \forall s \in[0,2],
\end{aligned}
$$

where the first equality follows from definition (26c), the second inequality from the fact that the $L^{2}\left(\Gamma_{i}\right)$-projection is optimal, and the third from estimate (25). Dividing expression (27) by $\|\psi\|_{s, \Gamma_{i}}$ and taking the supremum over all $\psi \in H_{0}^{s}\left(\Gamma_{i}\right) \backslash\{0\}$ yields, by definition (5),

$$
\left\|g-P_{h}^{\gamma_{i}} g\right\|_{-s, \Gamma_{i}} \leq C h^{s}\|g\|_{0, \Gamma_{i}} \quad \forall s \in[0,2] .
$$

We will also need the inverse estimate

$$
\left\|v_{h}\right\|_{s, \Gamma} \leq C h^{-s}\left\|v_{h}\right\|_{0, \Gamma}, \quad s \in[0,1] .
$$

In contrast to an approximation estimate like (25), the inverse estimate requires quasiuniform mesh refinements [3, Definition 4.4.13]. That is, the quotient between the largest and smallest diameter of any triangle or line segment should stay uniformly bounded as the mesh is refined. Brenner and Scott [3, Theorem 4.5.11], for instance, prove inverse estimates for integral $s$ and domains in $\mathbb{R}^{n}$. Local bi-Lipschitz change of variables, partition of unity, and operator interpolation extend these estimates to estimate (29).

4. The variational form. We will make precise the idea of a solution to the Poisson equation based on the Lions-type variational expression (3). Let us define a linear form $G: L^{2}(\Omega) \rightarrow \mathbb{R}$ by the following procedure.

1. Given an element $v \in L^{2}(\Omega)$, find $z \in H_{0}^{1}(\Omega)$ such that

$$
\int_{\Omega} \nabla z \cdot \nabla w d x=\int_{\Omega} v w d x \quad \forall w \in H_{0}^{1}(\Omega) .
$$

2. From $v$ and $z$, find $\lambda \in L^{2}(\Gamma)$ such that

$$
-\int_{\Gamma} \lambda \phi d \Gamma=\int_{\Omega} v \phi d x-\int_{\Omega} \nabla z \cdot \nabla \phi d x \quad \forall \phi \in H^{1}(\Omega) .
$$

3. Set, for given $g \in L^{2}(\Gamma)$ and $f \in L^{2}(\Omega)$, uniquely associated with each $G$,

$$
G(v)=-\int_{\Gamma} g \lambda d \Gamma+\int_{\Omega} f z d x .
$$

TheOREM 4.1. The form $G$ is a bounded linear functional on $L^{2}(\Omega)$.

Proof. From Theorem 3.2 it follows that $\lambda$ and the boundary integral involved in the definition of $G$ are well defined for each $g \in L^{2}(\Gamma)$. The form $G$ is linear in $v$ since $\lambda$ and $z$ are linear in $v$. From (30) follows the estimate

$$
\|z\|_{1} \leq C\|v\|_{-1} .
$$

Thus,

$$
\begin{aligned}
|G(v)| & \leq\|g\|_{0, \Gamma}\|\lambda\|_{0, \Gamma}+\|f\|_{-1}\|z\|_{1} \leq C\left(\|g\|_{0, \Gamma}\|\lambda\|_{0, \Gamma}+\|f\|_{-1}\|v\|_{-1}\right) \\
& \leq C\left(\|g\|_{0, \Gamma}+\|f\|_{0}\right)\|v\|_{0},
\end{aligned}
$$


where estimates (7), (33), and (16) are used in the first, second, and third inequality, respectively.

By Theorem 4.1 and Riesz representation, the following problem thus has a unique solution:

$$
\begin{aligned}
& \text { Find } u \in L^{2}(\Omega) \text { such that } \\
& \int_{\Omega} u v d x=G(v) \quad \forall v \in L^{2}(\Omega) .
\end{aligned}
$$

Problem (35) defines a weak solution to the Poisson problem (1), in which the boundary data $g$ needs only to be square-integrable. The price to pay for the reduced regularity requirement on $g$ is that $u \notin H^{1}(\Omega)$ in general and that the meaning of boundary condition $u=g$ will be weak; it will be satisfied only in a distributional sense acute a la Theorem 6.5 in Chapter 2 of Lions and Magenes [15, Volume 1].

Similar to solutions to (10), solutions to problem (35) have higher regularity than asked for.

TheOrem 4.2. For each $f \in L^{2}(\Omega)$ and $g \in L^{2}(\partial \Omega)$ associated with definition (32) of $G$, there exists an $\epsilon \in(0,1 / 2]$ so that the solution to problem (35) resides in $H^{s}(\Omega)$ for each $s \in[0, \epsilon)$.

Proof. First, note that $H_{0}^{s}(\Omega)=H^{s}(\Omega)$ for $s \in(0,1 / 2]$, which follows from the fact that $C_{0}^{\infty}(\Omega)$ is dense in $H^{s}(\Omega)$ for $s \in(0,1 / 2]$ [11, Theorem 1.4.2.4]. This equivalence allows the use of expression (6) to estimate $\|u\|_{s}$.

To estimate the boundary-integral term in the definition (32) of $G$, write the $L^{2}(\Gamma)$-norm of $\lambda$ as a sum over contributions from each polygonal surface and utilize estimate (16) in Theorem 3.2. It then follows that there exists an $\epsilon \in(0,1 / 2]$ such that, for each $s \in[0, \epsilon)$,

$$
\begin{aligned}
\left(\int_{\Gamma} g \lambda d \Gamma\right)^{2} & \leq\|\lambda\|_{0, \Gamma}^{2}\|g\|_{0, \Gamma}^{2}=\sum_{i=1}^{I}\|\lambda\|_{0, \Gamma_{i}}^{2}\|g\|_{0, \Gamma}^{2} \\
& \leq C \sum_{i=1}^{I}\|\lambda\|_{\epsilon-s, \Gamma_{i}}^{2}\|g\|_{0, \Gamma}^{2} \leq C\|v\|_{-s}^{2}\|g\|_{0, \Gamma}^{2} \quad \forall v \in L^{2}(\Omega) .
\end{aligned}
$$

We also estimate the second integral in the definition (32) of $G$ as

$$
\int_{\Omega} f z d x \leq\|f\|_{-1}\|z\|_{1} \leq\|f\|_{-1}\|z\|_{3 / 2+\epsilon} \leq C\|f\|_{-1}\|v\|_{-s} \quad \forall v \in L^{2}(\Omega),
$$

where the last inequality follows from Theorem 3.1. Equation (35), the definition (32) of $G$, and estimates (36) and (37) yield that, for some $\epsilon \in(0,1 / 2]$,

$$
\int_{\Omega} u v d x \leq C\|v\|_{-s}\left(\|g\|_{0, \Gamma}+\|f\|_{-1}\right) \quad \forall v \in L^{2}(\Omega), \forall s \in[0, \epsilon) .
$$

Dividing expression (38) by $\|v\|_{-s}$, taking the supremum over all $v \in L^{2}(\Omega) \backslash\{0\}$, and using property (6) yields the conclusion.

5. Numerical approximations. Recall the standard Galerkin approximation of the Poisson problem with inhomogeneous boundary data: If $g_{h} \in \gamma V_{h}$ approximates the boundary data, we solve the problem:

$$
\text { Find } u_{h} \in V^{h} \text { such that }\left.u_{h}\right|_{\Gamma}=g_{h} \text { and }
$$

$$
\int_{\Omega} \nabla u_{h} \cdot \nabla v_{h} d x=\int_{\Omega} f v_{h} d x \quad \forall v_{h} \in V_{0}^{h}
$$


The error in this approximation depends on how the boundary data is approximated. For homogeneous boundary data, $g_{h}=0$, standard error estimates and the regularity according to Theorem 3.1 yield that there exists an $\epsilon \in(0,1 / 2]$ such that

$$
\left\|u_{h}-u\right\|_{1} \leq C h^{1 / 2+\epsilon}\|f\|_{0}
$$

where the estimate holds for $\epsilon=1 / 2$ when the domain is convex.

Since $V^{h} \subset L^{2}(\Omega)$, we can apply a Galerkin approximation to problem (35):

$$
\text { Find } \widetilde{u}_{h} \in V^{h} \text { such that }
$$

$$
\int_{\Omega} \widetilde{u}_{h} v_{h} d x=G\left(v_{h}\right) \quad \forall v_{h} \in V^{h} .
$$

Subtracting (35) with $v=v_{h}$ from (41), we obtain

$$
\int_{\Omega}\left(\widetilde{u}_{h}-u\right) v_{h} d x=0 \quad \forall v_{h} \in V^{h},
$$

implying that the Galerkin approximation is optimal in $L^{2}(\Omega)$,

$$
\left\|\widetilde{u}_{h}-u\right\|_{0}=\inf _{v_{h} \in V^{h}}\left\|u-v_{h}\right\|_{0} \leq\left\|u-\Pi_{h} u\right\| .
$$

Approximation (41) is useless as a numerical method, however, since to compute $G\left(v_{h}\right)$, we need the exact solutions $z$ and $\lambda$ to problems (30) and (31) for each $v_{h} \in V^{h}$. A natural alternative is to use numerical approximations $z_{h}$ and $\lambda_{h}$ instead, which pertains to a modification of $G$-a so-called variational crime. For this, define the linear form $G_{h}: L^{2}(\Omega) \rightarrow \mathbb{R}$ as follows:

1. Given $v \in L^{2}(\Omega)$, find $z_{h} \in V_{0}^{h}$ such that

$$
\int_{\Omega} \nabla z_{h} \cdot \nabla w_{h} d x=\int_{\Omega} v w_{h} d x \quad \forall w_{h} \in V_{0}^{h} .
$$

2. From $v$ and $z_{h}$, compute $\lambda_{h} \in \gamma V^{h}$ such that

$$
-\int_{\Gamma} \lambda_{h} \phi_{h} d \Gamma=\int_{\Omega} v \phi_{h} d x-\int_{\Omega} \nabla z_{h} \cdot \nabla \phi_{h} d x \quad \forall \phi_{h} \in M^{h} .
$$

3. Set, given $g \in L^{2}(\Gamma)$ and $f \in L^{2}(\Omega)$,

$$
G_{h}(v)=-\int_{\Gamma} g \lambda_{h} d \Gamma+\int_{\Omega} f z_{h} d x .
$$

A second approximation to (35) is as follows:

$$
\begin{aligned}
& \text { Find } u_{h} \in V^{h} \text { such that } \\
& \int_{\Omega} u_{h} v_{h} d x=G_{h}\left(v_{h}\right) \quad \forall v_{h} \in V^{h} .
\end{aligned}
$$

At first glance, approximation (46) appears unreasonably costly to implement, since the computation of each component of the vector $G_{h}\left(v_{h}\right)$ requires the solution of (43) and (44)! However, a remarkable property of approximation (46), shown in Theorem 5.2, is its equivalence to the standard Galerkin approximation (39), provided that the $L^{2}(\Gamma)$ projection is used to approximate the inhomogeneous boundary 
conditions. Thus, in practical computations, approximation (46) can be implemented as a standard Galerkin approximation combined with $L^{2}$-projections of the boundary data. The equivalence of Theorem 5.2 is a consequence of the properties of the mapping $v_{h} \mapsto\left(z_{h}, \lambda_{h}\right)$ involved in the definition of $G_{h}$.

LEMMA 5.1. The mapping $v_{h} \mapsto\left(z_{h}, \lambda_{h}\right)$, defined by solving (43) and (44), is bijective as a mapping $V^{h} \rightarrow V_{0}^{h} \times \gamma V^{h}$. Moreover, the functions $v_{h}$, $z_{h}$, and $\lambda_{h}$ satisfy

$$
\int_{\Omega} v_{h} \psi_{h} d x=-\int_{\Gamma} \lambda_{h} \psi_{h} d \Gamma+\int_{\Omega} \nabla z_{h} \cdot \nabla \psi_{h} d x \quad \forall \psi_{h} \in V^{h} .
$$

Proof. Let $v_{h} \in V^{h}$ be given, and let $z_{h} \in V_{0}^{h}$ and $\lambda_{h} \in \gamma V^{h}$ be the unique solutions to (43) and (44) for $v=v_{h}$.

Expression (43) with $v=v_{h}$ can be written

$$
0=\int_{\Omega} v_{h} w_{h} d x-\int_{\Omega} \nabla z_{h} \cdot \nabla w_{h} d x \quad \forall w_{h} \in V_{0}^{h} .
$$

Adding (48) to (44) with $v=v_{h}$ yields

$$
-\int_{\Gamma} \lambda_{h} \phi_{h} d \Gamma=\int_{\Omega} v_{h}\left(\phi_{h}+w_{h}\right) d x-\int_{\Omega} \nabla z_{h} \cdot \nabla\left(\phi_{h}+w_{h}\right) d x
$$

for each $\phi_{h} \in M^{h}$ and each $w_{h} \in V_{0}^{h}$. Since $V^{h}=V_{0}^{h} \oplus M^{h}$, and since functions in $V_{0}^{h}$ vanish on $\Gamma$, it follows that $v_{h}, z_{h}$, and $\lambda_{h}$ are related through expression (47).

Conversely, let $z_{h} \in V_{0}^{h}$ and $\lambda_{h} \in \gamma V^{h}$ be given. Expression (47) defines an equation for $v_{h}$ corresponding to a square linear system with a positive-definite matrix. Equation (47) thus has a unique solution $v_{h} \in V^{h}$. The mapping $v_{h} \mapsto\left(z_{h}, \lambda_{h}\right)$ is thus bijective, since the mapping itself as well as its inverse are one-to-one.

With the aid of the mapping of Lemma 5.1, we can transfer between the "new" approximation (46) and the traditional (39), as follows.

THEOREM 5.2. The function $u_{h} \in V^{h}$ is a solution to problem (46) if and only if

$$
\begin{aligned}
& u_{h} \in V^{h} \text { such that } \\
& \begin{aligned}
\int_{\Omega} \nabla u_{h} \cdot \nabla z_{h} d x & =\int_{\Omega} f z_{h} d x \quad \forall z_{h} \in V_{0}^{h}, \\
u_{h} & =P_{h}^{\gamma} g \quad \text { on } \Gamma,
\end{aligned}
\end{aligned}
$$

where $P_{h}^{\gamma} g$ is the $L^{2}(\Gamma)$-projection of $g$ on $\gamma V^{h}$, that is,

$$
\begin{aligned}
& P_{h}^{\gamma} g \in \gamma V^{h} \text { such that } \\
& \int_{\Gamma} P_{h}^{\gamma} g r_{h} d \Gamma=\int_{\Gamma} g r_{h} d \Gamma \quad \forall r_{h} \in \gamma V^{h} .
\end{aligned}
$$

Proof. (i) Let $u_{h}$ be the solution to problem (50). Let $v_{h} \in V^{h}$ be given, and compute $z_{h} \in V_{0}^{h}$ and $\lambda_{h} \in \gamma V^{h}$ by solving (43) and (44) with $v=v_{h}$. By Lemma 5.1, $v_{h}, z_{h}$, and $\lambda_{h}$ are related through the expression

$$
-\int_{\Gamma} \lambda_{h} \psi_{h} d \Gamma+\int_{\Omega} \nabla z_{h} \cdot \nabla \psi_{h} d x=\int_{\Omega} v_{h} \psi_{h} d x \quad \forall \psi_{h} \in V^{h} .
$$


Choosing $\psi_{h}=u_{h}$, it follows that

$$
-\int_{\Gamma} \lambda_{h} u_{h} d \Gamma+\int_{\Omega} \nabla z_{h} \cdot \nabla u_{h} d x=\int_{\Omega} v_{h} u_{h} d x .
$$

Using (50a) to replace the second term in expression (52), we obtain

$$
\begin{aligned}
\int_{\Omega} u_{h} v_{h} d x & =-\int_{\Gamma} \lambda_{h} u_{h} d \Gamma+\int_{\Omega} f z_{h} d x=-\int_{\Gamma} \lambda_{h} P_{h}^{\gamma} g d \Gamma+\int_{\Omega} f z_{h} d x \\
& =-\int_{\Gamma} \lambda_{h} g d \Gamma+\int_{\Omega} f z_{h} d x=G_{h}\left(v_{h}\right),
\end{aligned}
$$

where we have used (50b) in the second equality, definition (51) of $P_{h}^{\gamma} g$ in the third, and definition (45) of $G_{h}$ in the fourth equality. Since $v_{h} \in V^{h}$ was arbitrary, we have shown that if $u_{h}$ solves (50), it also solves (46).

(ii) Conversely, let $u_{h}$ be the solution of problem (46), and let $\lambda_{h} \in \gamma V^{h}$ and $z_{h} \in V_{0}^{h}$ be given. By Lemma 5.1, there is a unique $v_{h} \in V^{h}$ satisfying

$$
\int_{\Omega} v_{h} \psi_{h} d x=-\int_{\Gamma} \lambda_{h} \psi_{h} d \Gamma+\int_{\Omega} \nabla z_{h} \cdot \nabla \psi_{h} d x \quad \forall \psi_{h} \in V^{h}
$$

Choosing $\psi_{h}=u_{h}$, we find

$$
-\int_{\Gamma} \lambda_{h} u_{h} d \Gamma+\int_{\Omega} \nabla z_{h} \cdot \nabla u_{h} d x=\int_{\Omega} v_{h} u_{h} d x .
$$

Since $u_{h}$ is a solution of problem (46), the right-hand side of expression (55) satisfies

$$
\int_{\Omega} v_{h} u_{h} d x=-\int_{\Gamma} g \widehat{\lambda}_{h} d \Gamma+\int_{\Omega} f \widehat{z}_{h} d x
$$

where $\widehat{z}_{h}$ and $\widehat{\lambda}_{h}$ are the solutions to (43) and (44) with $v=v_{h}$. However, since by Lemma 5.1, $\widehat{z}_{h}$ and $\widehat{\lambda}_{h}$ are uniquely defined by $v_{h}$, we have $\widehat{z}_{h}=z_{h}$ and $\widehat{\lambda}_{h}=\lambda_{h}$. Substituting expression (56) into expression (55), we obtain

$$
\begin{aligned}
\int_{\Omega} \nabla u_{h} \cdot \nabla z_{h} d x & =\int_{\Gamma} \lambda_{h}\left(u_{h}-g\right) d \Gamma+\int_{\Omega} f z_{h} d x \\
& =\int_{\Gamma} \lambda_{h}\left(u_{h}-P_{h}^{\gamma} g\right) d \Gamma+\int_{\Omega} f z_{h} d x
\end{aligned}
$$

where in the last equality we introduce $P_{h}^{\gamma} g \in \gamma V^{h}$ as the solution of (51). Since the choices of $z_{h}$ and $\lambda_{h}$ were arbitrary, it follows that both (50a) and the boundary condition (50b) must be satisfied.

The difference between problems (41) and (46) lies in the use of an approximated linear form $G_{h}$ in problem (46). It is therefore crucial to analyze the error that the use of $G_{h}$ introduces. Lemma 5.4, which estimates $\left(G-G_{h}\right)$, needs the following discrete extension result.

Lemma 5.3. There exists a $C>0$, independent of $h>0$, such that for each $g_{h} \in \gamma V^{h}, a u_{h} \in V^{h}$ exists satisfying $\left.u_{h}\right|_{\Gamma}=g_{h}$ and

$$
\left\|u_{h}\right\|_{1} \leq C\left\|g_{h}\right\|_{1 / 2, \Gamma} .
$$


Proof. Let $g_{h} \in \gamma V^{h}$ be given. The functions in $\gamma V^{h}$ are continuous and piecewise linear on the boundary, so $\gamma V^{h} \subset H^{1 / 2}(\Gamma)$ (in fact, $\gamma V^{h} \subset H^{1}(\Gamma)$ ). By Theorem 1.5.1.3 of Grisvard [11], the trace map $\gamma: H^{1}(\Omega) \rightarrow H^{1 / 2}(\Omega)$ has a right continuous inverse $E$. The Scott and Zhang interpolator $\Pi_{h}$, discussed in section 3.3, continuously maps functions in $H^{1}(\Omega)$ into $V^{h}$. Composing $\Pi_{h}$ and $E$, we define $u_{h} \in V^{h}$ such that $u_{h}=\Pi_{h} E g_{h}$. Note that $\gamma u_{h}=g_{h}$ since the Scott and Zhang interpolator preserves piecewise-polynomial boundary conditions. Moreover, since both $E$ and $\Pi_{h}$ are continuous, we find that

$$
\left\|u_{h}\right\|_{1} \leq C\left\|g_{h}\right\|_{1 / 2, \Gamma}
$$

Remark 1. Similar results are reported in the estimate (5.5) of Scott and Zhang [17] and in Lemma 11 of Gunzburger and Hou [13].

LEMma 5.4. Assume a quasi-uniform triangulation, characterized by the mesh parameter $h$, of the polyhedral (or polygonal) domain $\Omega$ having a Lipschitz boundary. There are an $\epsilon \in(0,1 / 2]$ and $a C>0$ such that, given $g \in L^{2}(\Gamma)$ and $f \in L^{2}(\Omega)$, the linear forms $G$ and $G_{h}$, defined in expressions (32) and (45), satisfy, for each $h>0$,

$$
\left(G-G_{h}\right)(v) \leq C\left(h^{\epsilon}\|g\|_{0, \Gamma}+h^{1 / 2+\epsilon}\|f\|_{-1}\right)\|v\|_{0} \quad \forall v \in L^{2}(\Omega) .
$$

Proof. Let $v \in L^{2}(\Omega)$ be given, and let $z$ and $\lambda$ be the solutions to (30) and (31) associated with the given $v$. Likewise, let $z_{h}$ and $\lambda_{h}$ be the solutions to (43) and (44) associated with $v$. By definitions (32) and (45), we find that

$$
\begin{aligned}
\left(G-G_{h}\right)(v) & =-\int_{\Gamma} g\left(\lambda-\lambda_{h}\right) d \Gamma+\int_{\Omega} f\left(z-z_{h}\right) d x \\
= & -\int_{\Gamma}\left(\lambda-\lambda_{h}\right) P_{h}^{\gamma} g d \Gamma-\int_{\Gamma} \lambda\left(g-P_{h}^{\gamma} g\right) d \Gamma+\int_{\Omega} f\left(z-z_{h}\right) d x
\end{aligned}
$$

introducing $P_{h}^{\gamma} g$, the $L^{2}(\Gamma)$ projection of $g$ on $\gamma V^{h}$, defined as in (26b).

We will estimate each of the terms in the right-hand side of expression (57), starting with the first. Let $\varphi_{h} \in \gamma V^{h}$ be given. Choose $\phi_{h} \in V_{h}$ so that $\left.\phi_{h}\right|_{\Gamma}=\varphi_{h}$ and so that the estimate of Lemma 5.3 is satisfied. From (31) and (44) it follows that

$$
\begin{aligned}
-\int_{\Gamma}( & \left.\lambda-\lambda_{h}\right) \varphi_{h} d \Gamma=-\int_{\Omega} \nabla\left(z-z_{h}\right) \cdot \nabla \phi_{h} d x \\
& \leq\left\|z_{h}-z\right\|_{1}\left\|\phi_{h}\right\|_{1} \leq C\left\|z_{h}-z\right\|_{1}\left\|\varphi_{h}\right\|_{1 / 2, \Gamma} \\
& \leq C\left\|z_{h}-z\right\|_{1} h^{-1 / 2}\left\|\varphi_{h}\right\|_{0, \Gamma} \leq C h^{1 / 2+\epsilon}\|v\|_{0} h^{-1 / 2}\left\|\varphi_{h}\right\|_{0, \Gamma} \\
& =C h^{\epsilon}\|v\|_{0}\left\|\varphi_{h}\right\|_{0, \Gamma},
\end{aligned}
$$

where the second inequality follows from Lemma 5.3 and the third from inverse estimates (29) (the inverse estimate needs the assumption of quasi-uniform mesh refinements); expression (40) yields the existence of an $\epsilon \in(0,1 / 2]$ such that the fourth inequality holds.

Next we estimate the second term in the right-hand side of expression (57) as 
follows:

$$
\begin{aligned}
\int_{\Gamma} \lambda\left(g-P_{h}^{\gamma} g\right) d \Gamma & =\sum_{i=1}^{I} \int_{\Gamma_{i}} \lambda\left(g-P_{h}^{\gamma} g\right) d \Gamma \leq \sum_{i=1}^{I}\|\lambda\|_{\epsilon, \Gamma_{i}}\left\|g-P_{h}^{\gamma} g\right\|_{-\epsilon, \Gamma_{i}} \\
& \leq C\|v\|_{0} \sum_{i=1}^{I}\left\|g-P_{h}^{\gamma} g\right\|_{-\epsilon, \Gamma_{i}} \leq C\|v\|_{0}\left\|g-P_{h}^{\gamma} g\right\|_{-\epsilon, \Gamma} \\
& \leq C h^{\epsilon}\|v\|_{0}\|g\|_{0, \Gamma},
\end{aligned}
$$

where the second, third, and fourth inequalities use estimates (16), (9), and (28), respectively. The third term in the right-hand side of expression (57) is estimated by expression (40),

$$
\int_{\Omega} f\left(z-z_{h}\right) d x \leq\|f\|_{-1}\left\|z-z_{h}\right\|_{1} \leq C h^{1 / 2+\epsilon}\|f\|_{-1}\|v\|_{0} .
$$

Substituting estimates (58) (with $\varphi_{h}=P_{h}^{\gamma} g$ ), (59), and (60) into expression (57) yields the required estimate

$$
\begin{aligned}
\left(G-G_{h}\right)(v) & \leq C\left(h^{\epsilon}\left\|P_{h}^{\gamma} g\right\|_{0, \Gamma}+h^{\epsilon}\|g\|_{0, \Gamma}+h^{1 / 2+\epsilon}\|f\|_{-1}\right)\|v\|_{0} \\
& \leq C\left(h^{\epsilon}\|g\|_{0, \Gamma}+h^{1 / 2+\epsilon}\|f\|_{-1}\right)\|v\|_{0},
\end{aligned}
$$

where in the second inequality we have used the bound $\left\|P_{h}^{\gamma} g\right\|_{0, \Gamma} \leq\|g\|_{0, \Gamma}$ that holds for an $L^{2}$-projection.

The final result of this article is that the solution to (46) converges to the solution of (35) at a rate that depends, through $\epsilon$, on the shape of the domain, where $\epsilon=1 / 2$ corresponds to a convex domain.

THEOREM 5.5. Assume a quasi-uniform triangulation, characterized by the mesh parameter $h$, of the polyhedral (or polygonal) domain $\Omega$ having a Lipschitz boundary. There are an $\epsilon \in(0,1 / 2]$ and $a C>0$ such that, given $g \in L^{2}(\Gamma)$ and $f \in L^{2}(\Omega)$, the solutions $u$ and $u_{h}$ to problems (35) and (46) satisfy, for each $h>0$,

$$
\left\|u-u_{h}\right\|_{0} \leq C\left(h^{s}\|u\|_{s}+h^{\epsilon}\|g\|_{0, \Gamma}+h^{1 / 2+\epsilon}\|f\|_{-1}\right)
$$

for each $s \in[0, \epsilon)$.

Proof. The solution error may be decomposed as $u-u_{h}=\left(u-\widetilde{u}_{h}\right)+\left(\widetilde{u}_{h}-u_{h}\right)$, where $\widetilde{u}$ is the solution to problem (41). Thus,

$$
\left\|u-u_{h}\right\|_{0} \leq\left\|u-\widetilde{u}_{h}\right\|+\left\|\widetilde{u}_{h}-u_{h}\right\| .
$$

By estimate (42), approximation property (24b), and Theorem (4.2), there exists an $\epsilon \in(0,1 / 2]$ so that

$$
\left\|u-\widetilde{u}_{h}\right\|_{0} \leq C h^{s}\|u\|_{s} \quad \forall s \in[0, \epsilon) .
$$

Equations (41) and (46) yield that

$$
\int_{\Omega}\left(\widetilde{u}_{h}-u_{h}\right) v_{h} d x=G\left(v_{h}\right)-G_{h}\left(v_{h}\right) \quad \forall v_{h} \in V^{h},
$$


so choosing $v_{h}=\widetilde{u}_{h}-u_{h}$ means that

$$
\left\|\widetilde{u}_{h}-u_{h}\right\|_{0}^{2}=\left(G-G_{h}\right)\left(\widetilde{u}_{h}-u_{h}\right) .
$$

Using Lemma 5.4 in expression (63) implies that

$$
\left\|\widetilde{u}_{h}-u_{h}\right\|_{0} \leq C\left(h^{\epsilon}\|g\|_{0, \Gamma}+h^{1 / 2+\epsilon}\|f\|_{-1}\right) .
$$

Substituting estimates (62) and (64) into expression (61) provides the required estimate.

Remark 2. Theorem 5.5 only provides convergence rates for boundary data in $L^{2}(\Gamma)$. By the equivalence proven in Theorem 5.2, smoother data will improve the convergence rate, since error estimates for the standard approach then apply. Fix, Gunzburger, and Peterson [6], French and King [7, 8], and Bramble and King [2] prove various error estimates that apply for smoother data.

Acknowledgments. I am grateful to my former advisor Roland Glowinski, who once told his surprised student that the ordinary finite-element approximation converges, although in a weaker sense, also for rough data. This comment sparked an interest, eventually leading to the investigations reported here. For careful readings of the manuscript, which uncovered many issues in need of improvement, I owe much to Ridgway Scott. I also thank Max Gunzburger, Steven Hou, and Christer Kiselman for helpful discussions.

\section{REFERENCES}

[1] I. BABUŠKA, Error-bounds for finite element method, Numer. Math., 16 (1971), pp. 322-333.

[2] J. H. Bramble and J. T. KIng, A robust finite element method for nonhomogeneous Dirichlet problems in domains with curved boundaries, Math. Comp., 63 (1994), pp. 1-17.

[3] S. C. Brenner And L. R. Scott, The Mathematical Theory of Finite Element Methods, Springer-Verlag, New York, 1994.

[4] M. Chevalier, M. Högberg, M. Berggren, and D. S. Henningson, Linear and nonlinear optimal control in spatial boundary layers, in Proceedings of the AIAA 3rd Theoretical Fluid Mechanics Meeting, St. Louis, MO, AIAA Paper 2002-2755, 2002.

[5] P. G. Ciarlet, The Finite Element Method for Elliptic Problems, North-Holland, Amsterdam, 1978.

[6] G. J. Fix, M. D. Gunzburger, and J. S. Peterson, On finite element approximations of problems having inhomogeneous essential boundary conditions, Comput. Math. Appl., 9 (1983), pp. 687-700.

[7] D. A. French And J. T. KIng, Approximation of an elliptic control problem by the finite element method, Numer. Funct. Anal. Optim., 12 (1991), pp. 299-314.

[8] D. A. French AND J. T. KING, Analysis of a robust finite element approximation for a parabolic equation with rough boundary data, Math. Comp., 60 (1993), pp. 79-104.

[9] R. Glowinski And J. L. Lions, Exact and approximate controllability for distributed parameter systems, Acta Numer., 1994, pp. 269-378.

[10] R. Glowinski And J. L. Lions, Exact and approximate controllability for distribtuted parameter systems, Acta Numer., 1995, p. 159-333.

[11] P. Grisvard, Elliptic Problems in Non-Smooth Domains, Pitman, London, 1985.

[12] P. Grisvard, Singularities in Boundary Value Problems, Masson, Paris, and Springer-Verlag, Berlin, 1992.

[13] M. D. Gunzburger And S. L. Hou, Treating inhomogeneous essential boundary conditions in finite element methods and the calculation of boundary stresses, SIAM J. Numer. Anal., 29 (1992), pp. 390-424.

[14] J. L. Lions, Optimal Control of Systems Governed by Partial Differential Equations, SpringerVerlag, Berlin, 1971. 
[15] J. L. Lions And E. Magenes, Non-Homogeneous Boundary Value Problems and Applications, Springer-Verlag, Berlin, 1972.

[16] P. J. Schmid And D. S. Henningson, Stability and Transition in Shear Flows, Appl. Math. Sci. 142, Springer-Verlag, New York, 2000.

[17] L. R. Scott And S. Zhang, Finite element interpolation of nonsmooth functions satisfying boundary conditions, Math. Comp., 54 (1990), pp. 483-493.

[18] K. Yosida, Functional Analysis, Springer-Verlag, Berlin, 1980. 